\title{
Estramustine Phosphate Sodium
}

National Cancer Institute

\section{Source}

National Cancer Institute. Estramustine Phosphate Sodium. NCI Thesaurus. Code C480.

The orally available disodium salt, monohydrate, of estramustine phosphate, a synthetic molecule that combines estradiol and nornitrogen mustard through a carbamate link. Estramustine and its major metabolite estramustine bind to microtubule-associated proteins (MAPs) and tubulin, thereby inhibiting microtubule dynamics and leading to anaphase arrest in a dose-dependent fashion. This agent also exhibits anti-androgenic effects. 